\title{
EELS Characterization of Tin Oxides
}

\author{
M.S. Moreno* and R.F. Egerton ${ }^{* *}$ \\ * Centro Atómico Bariloche, 8400 San Carlos de Bariloche, Argentina \\ ** Department of Physics, University of Alberta, Edmonton, Canada T6G 2J1
}

Tin oxides are technologically important materials with several applications, for example as semiconductor and gas sensing devices, solar cells, catalysis and lithium ion batteries. Their uses involve bulk and nanostructured materials and thin films. Many synthesis routes involve changes in the oxidation state of tin, so there is a need for distinguishing between the two stable oxides $\left(\mathrm{SnO}_{2}\right.$ and $\mathrm{SnO}$ ) at high spatial resolution, by acquiring information on the elemental composition and chemical state. For these purpose many low-energy (surface) spectroscopies fail. Here we explore the capability of electron energy-loss spectroscopy (EELS) carried out in a conventional TEM.

We have used commercial (Aldrich) powders of both oxides. Energy-loss spectra were measured by using a Gatan 666 spectrometer attached to a JEOL 2010 microscope operated at $200 \mathrm{kV}$. Because $\mathrm{SnO}$ has been reported to be a beam sensitive material [1] we verified that out specimen remained undamaged at the dose used for the experiments, by monitoring the electron diffraction pattern and the energy-loss spectrum for possible structural and/or electronic changes.

Typical spectra are shown in Figure 1, where it can be seen that the O-K edge is completely overlapped with the $\mathrm{M}_{4}$ and $\mathrm{M}_{5}$ delayed edges of tin. Despite this difficulty, it is possible to distinguish clear differences in the near-edge structure (ELNES) that can be used as a fingerprint of each phase. Some minor differences are also observed.

To correlate ELNES spectral features with Sn and O contributions, we have carried out full multiple scattering calculations using the FEFF 8.20 program [2]. Potentials were calculated self-consistently. We studied the effect of several parameters on the simulated ELNES and concluded that a good choice is the use of non-overlapping muffin-tin radii and the Hedin-Lundqvist self-energy exchange correlation potential. We also considered the presence final-state effects resulting from the core-level excitation. It appears that the ELNES of both phases can be best calculated with the presence of a core hole. The calculations shown in Figure1 indicate that Sn makes the main contribution below $530 \mathrm{eV}$ but the pronounced structure at $530-540 \mathrm{eV}$ is mainly due to oxygen and is entirely different in both phases. Other features represent a mixture of both contributions [3].

[1] M.S.Moreno, A.Varela, L.C.Otero-Díaz, Phys. Rev. B 56 (1997) 5186

[2] A.L.Ankudinov, B.Ravel, J.J.Rehr, S.D.Conradson, Phys.Rev.B 58 (1998)7565

[3] MSM acknowledge the financial support of Fundación Antorchas through the grant 14022-100. Partial financial support of CONICET-Argentina is also acknowledged. 

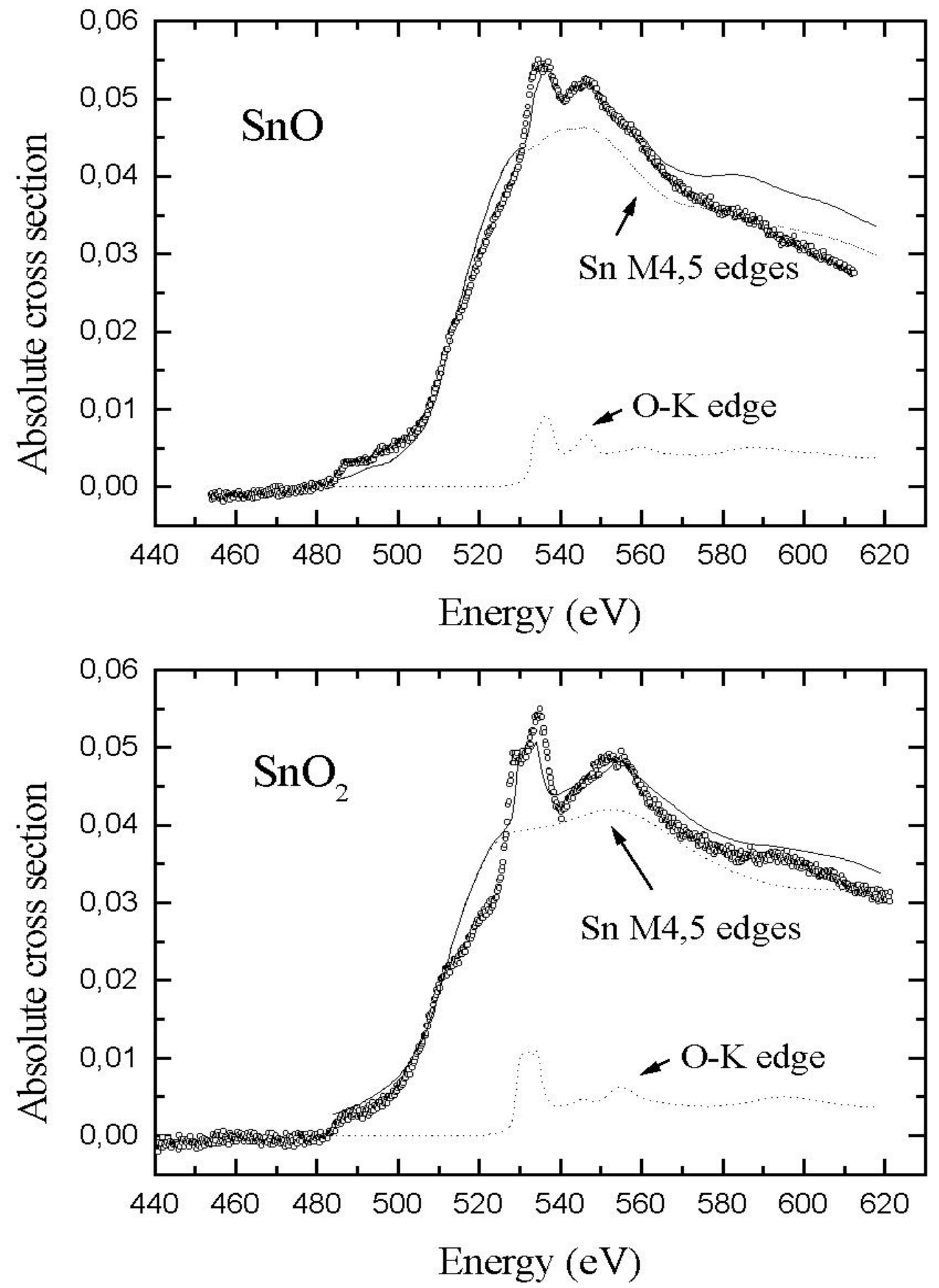

Figure 1. Comparison of EELS data (open circles) and calculations (solid line). Contributions of both tin and oxygen edges are also shown (as dotted lines). Upper panel: $\mathrm{SnO}$, experimental resolution $2.6 \mathrm{eV}$. Lower panel: $\mathrm{SnO}_{2}$, experimental resolution $1.8 \mathrm{eV}$. 